\title{
Mythologies du réel : récits de fiction et discours critiques
}

Bruno Blanckeman

\section{OpenEdition}

1 Journals

Édition électronique

URL : http://journals.openedition.org/recherchestravaux/422

DOI : 10.4000/recherchestravaux.422

ISSN : 1969-6434

Éditeur

UGA Éditions/Université Grenoble Alpes

Édition imprimée

Date de publication : 20 décembre 2010

Pagination : 69-76

ISBN : 978-2-84310-187-8

ISSN : 0151-1874

Référence électronique

Bruno Blanckeman, "Mythologies du réel : récits de fiction et discours critiques », Recherches \&

Travaux [En ligne], 77 | 2010, mis en ligne le 20 août 2012, consulté le 08 septembre 2020. URL : http:// journals.openedition.org/recherchestravaux/422 ; DOI : https://doi.org/10.4000/recherchestravaux. 422 
Bruno BLANCKEMAN

Université Paris 3-Sorbonne nouvelle

\section{Mythologies du réel : récits de fiction et discours critiques}

Que sont Les Mythologies devenues en cette première décennie du XXI ${ }^{\mathrm{e}}$ siècle? Cette matière idéologique présente à l'état de produits standards que pointait voilà un demi-siècle Roland Barthes, ce politiquement correct à portée d'oreille et de bouche, revêtu des costumes d'époque... Au débotté, et sans prétention à l'exhaustivité, aujourd'hui : des nourritures, sacralisées, non plus le beefsteak frites mais le bio; des pratiques, hygiénistes, le jogging, pour qui aime courir à droite, à petites foulées libérales, ou le vélib’, pour qui préfere rouler à gauche, en tendance rose-bobo; des objets, qui collent comme naturellement à la peau tels ces antitranspirants, ne plus dire «déodorants", qui empêchent de se faire suer quand bien même on marne douze heures d'affilée, donc constituent une incitation au corps, un flux irrépressible au productivisme d'entreprise; des catégories qui fascinent, non plus le «sang bleu» mais les people, qui témoignent de la puissance des médias à accréditer de toutes pièces une réalité de pure fiction, transcatégorie socioculturelle de bric et de broc qui tient de l'inventaire à la Prévert, le raton laveur en moins, acteurs, têtes couronnées, sportifs, politiques, bateleurs de télévision; des mots ou des métaphores, aussi, le «bien évidemment» qui scande nos discours, naturalisant les propos les plus lestés d'intentions, ou encore, dans la presse, la tendance à employer de façon métaphorique le mot «logiciel» avec un sens qui tantôt recouvre celui de programme tantôt celui d'idéologie, devenu lui-même indésirable.

Bien des écrivains conçoivent leur métier comme la mise en jeu de ces mythologies. Écrire, c'est engager un rapport au réel qui ne se satisfait pas de leurs injonctions, fussent-elles éphémères. C'est en cela qu'on peut parler d'un «devenir roman» des Mythologies: non pas en cherchant à relever d'hypothétiques allusions à tel ou tel de ses chapitres dans tel ou tel roman ultérieur, mais 
en montrant comment, à partir des années I980, au sortir de l'ère du soupçon, l'essai de Barthes constitue une référence pour des écrivains désireux de représenter le monde dans lequel ils vivent par le biais de la fiction, et qui sont ainsi amenés à interroger de pair la catégorie du réel et la pratique du roman. Hasard du temps ou nécessité du système, ces écrivains, qu’ils aient nom Jean Echenoz ou Pierre Bergounioux, François Bon ou Pascal Quignard, appartiennent à une génération qui a fait ses classes à l'école du structuralisme. Resituées dans cette histoire, les Mythologies de Barthes constituent, sinon une source d'inspiration directe, du moins un repère décisif parce que le livre concilie, depuis un concentré narratif qui lui est propre, un regard moraliste renouvelé (La Bruyère à France Observateur) et un discours sémioticien en souplesse (l'acuité des signes sans le plomb du jargon). Cette double teneur caractérise aussi la littérature romanesque la plus ambitieuse du dernier vingtième siècle, voire du premier vingt et unième. Seul l'en sépare le spray marxisant qui fige encore, par endroits, la démarche des Mythologies et auquel elle ne recourt plus pour mettre en plis son propre discours.

Le problème soulevé concerne la capacité propre à la littérature au présent de donner sens au présent, c'est-à-dire d'appréhender, de configurer, de symboliser le réel, et cela en se détachant des stéréotypes et imageries qui en figent la connaissance et le gonflent d'évidence. L'écriture littéraire serait celle de tous les détachements, qu'elle affectionne à cet effet l'humour discret d'un Echenoz, l'expressionnisme fantastique d'un Antoine Volodine, les expansions baroques d'une Sylvie Germain, la concision axiomatique d'une Annie Ernaux. La qualité élémentaire de leurs récits tient à leur capacité commune de limiter ce que Roland Barthes appelait le degré endoxique du réel, les opinions et croyances convenues qui en empoissent la connaissance. Ces écrivains restituent le réel au travail du sens depuis des stéréotypes d'époque ou des évidences validées par le temps que bon nombre de romans se contentent de répéter. Leur travail n'est pas de l'ordre de la consignation : ils ne prélèvent pas des bribes de réalité qui préexisteraient au geste même d'écrire, ils travaillent le réel, en élaborent des représentations orientées à partir d'expériences sélectives, inventant de la sorte un réel critique de lui-même.

Sans doute convient-il de creuser d'emblée la problématique en envisageant alors un triple processus de mythologisation : le premier concerne les représentations communes du réel (mythologies ordinaires); le deuxième, les représentations littéraires du réel - distinction de taille puisqu'elle peut fonctionner comme critère de littérarité, invitant à dissocier une littérature de l'adhésion, qui colle aux mythologies ordinaires, et une littérature du détachement, qui les fait tomber; le troisième, les représentations critiques de la littérature elle-même, une mythologie du roman au présent, qui se fixe 
en priorité sur la question de son rapport au réel. En réponse à l'affirmation selon laquelle, depuis les années I980, il n'y aurait plus d'écrivains en France ni de sens de la fiction digne de ce nom, certains critiques et écrivains développent la théorie du retour. La littérature au présent se démarquerait de la modernité en cela qu'elle retrouverait le réel et en aurait fini avec une pratique d'elle-même à l'état pur. Elle serait ainsi marquée par un retour au et du réel, au et du sujet, au et du sens, à et de la fiction, retour sanctifié qui succède au temps des écarts hérétiques - comprendre, à celui de la modernité dans ses versions les plus radicales, qui se sont développées entre le milieu des années 1950 et celui des années 1970. Cette mythologie du discours critique, je lui attribuerai bien volontiers le nom de "polnareffisation" de la littérature : trente-cinq ans après avoir été congédié, le bon vieux roman revient, l'idole des foules fait son come-back à l'identique, même répertoire, même musique, même public, un peu vieilli certes, mais enfin, chirurgie plastique aidant, le charme opère encore ${ }^{\mathrm{I}}$. L'exemple le plus significatif de cette mythologie du discours critique, qui enchâsse par ses implications mêmes les deux autres ordres de mythologies, littéraires et ordinaires, est aussi celui qui met en cause directement... Roland Barthes et à travers lui, au nom du principe d'évidence et du bon gros bon sens, toute littérature qui envisage le réel à même sa production langagière, scripturale et idéologique :

Le monde revient. Et c'est la meilleure des nouvelles. N'aura-t-il pas été le grand absent de la littérature française? Le monde, le sujet, le sens, l'histoire, le «référent» : pendant des années, ils auront été mis entre parenthèses par les maitres penseurs, inventeurs d'une littérature sans autre objet qu'elle-même, faisant, comme il se disait alors, «sa propre critique dans le mouvement même de son énonciation $»^{2}$.

Les écritures contemporaines, du moins les plus attachantes d'entre elles, me semblent témoigner d'un ordre de résolutions plus complexe dans lequel le sens du pur - tout ce qui en elles entretient le réflexe autoscopique - et celui de l'impur - tout ce qui en elles relance la visée hétéroscopique - ne cessent de se mettre respectivement en perspective. Celles qui éprouvent aujourd'hui le réel avec le plus d'intensité circonscrivent un champ d'expériences données, en instituent des configurations signifiantes, mais incluent comme l'une de

I. Le chanteur-compositeur vedette Michel Polnareff s'exile aux États-Unis en 1973. En 2007, pour la première fois depuis son départ, il remonte sur scène à Paris pour un spectacle dans lequel il reprend tous ses succès d'antan.

2. J. Rouaud, M. Le Bris, Le Monde du I6 mars 2007, présentation de l'essai Pour une littérature-monde, Gallimard, 2007. Pour une étude critique détaillée des débats qu’il soulève, entre autres les débats sur la notion de francophonie, on se reportera au numéro 76 de Recherches et Travaux, juillet 20I0, "La littérature-monde : retour sur une polémique» (articles de Cl. Coste, V. Porra, C. François). 
ses données leur implication dans le système figuratif ainsi élaboré, assumant leur part de responsabilité esthétique, dans ce qui s'apparente moins à une représentation mimétique du réel qu’à une délibération critique autour du réel, c'est-à-dire une réflexion sur les champs qu'il recouvre et les tensions qui le sous-tendent. De l'oscillation ainsi ménagée entre un mode d'instanciation référentielle et un système de détermination tropologique, entre une suite d'indicateurs sémiotiques et un jeu de combinatoires formelles, résultent les imaginaires variables d'un réel inventé de toutes pièces, sur fond de réalités attestées de toute vie. J'en donnerai plusieurs séries d'exemples qui attestent de l'étendue des possibles en matière de parades antimythologiques et représentent un héritage, parfois revendiqué, souvent libre de tout affichage, de la démarche de Roland Barthes.

Première d'entre elles, l'œuvre d'un écrivain qui exprime ouvertement sa dette à l'égard de Barthes : Jean-Philippe Toussaint. Dans La Télévision ${ }^{3}$, l'écrivain, décrivant un poste de télévision, détaille les processus de composition des images et met en perspective les phénomènes de dédoublement du réel. À des états de réalité premiers ramenés au statut de référents modelables à l'infini se mélangent les états d'une réalité seconde, d'autant plus inquiétante qu'elle exclut moins celui qui en est le spectateur qu' elle ne l'absorbe, le consomme, le digère, l'excrète. Il en résulte la vision d'une humanité en partie désactivée, ramenée au statut de matière molle. Le personnage-narrateur, comme distrait de lui-même par ce qui était censé le divertir, tente par le sevrage de préserver son statut d'agent, au gré de situations élémentaires - relation de couple, échanges sociaux, travaux domestiques, écriture - toutes également compromises. Les deux romans suivants - le diptyque Faire l'amour/Fuir ${ }^{4}$ - poursuivent cette interrogation minutieuse des envers du décor technologiques, cette investigation par l'humour des envers de la civilisation high-tech qui consiste à partir des objets fétiches de notre temps - les modes de transmission électroniques dans un cas, la téléphonie mobile dans un autre - pour en sonder les pervers effets spectraux, à tous les sens de l'adjectif. Faire l'amour adapte la carte du tendre à l'ère du virtuel : quels sont les chemins d'aimer, leur centre et leurs périphéries, leurs clairières et leurs ornières, en un temps où la planète entière est accessible au moyen d'un message sur ordinateur, mais où cette hyperproximité voue au détachement, à la perte du contact sensible et de la matière tangible, à l'invalidation des pôles effectifs de la conscience - le temps et ses fuseaux, l'espace et ses réseaux'? Jean-Philippe Toussaint superpose deux lignes de fiction : la première, héritée de la métaphysique pascalienne, invite

3. J.-Ph. Toussaint, La Télévision, Minuit, 1997.

4. Id., Faire l'amour, Minuit, 2002; Fuir, Minuit, 2005.

5. Id., Faire l'amour, op. cit. On se reportera par exemple aux pages I24-I26, I73-I74. 
à mesurer la relativité des repères humains à l'infini des espaces cosmiques dans lesquels ils s'abyment ${ }^{6}$; la seconde, comme un métarécit technologique, confronte ces mêmes repères à leur actuelle indistinction : le vrai/le faux, le réel/ le virtuel, la matière/l'image?.

Autre série d'exemples possibles : à côté des œuvres déjà installées en littérature, certains auteurs tout en codes, brillants et joueurs, surfant sur la vague souvent éphémère de la reconnaissance médiatique. Frédéric Beigbeder, Benoît Duteurtre, Jean-Charles Masserra, Jérôme Mauche : en commun, une volonté d'épingler des états d'actualité contemporaine majeurs depuis des éclats de langue type dominants, celle de la communication et de la pub (Beigbeder, I4 euros $^{8}$ ) ou de l'entreprise et de la macroéconomie mondialisée (Masserra, Amour, gloire et Cac 409 , Mauche, La Loi des rendements décroissants $\left.^{\mathrm{I}}\right)$. Parti pris ou manque de moyens? Ces auteurs sont toutefois moins des faiseurs d'œuvres que des capteurs de symptômes. À ce jour aucun n'a voulu ou su concevoir des dispositifs littéraires qui fonctionneraient comme une machinerie de combat (Les Mythologies, puissante machine à recerveler), fût-ce à titre de cheval de Troie (Les Choses, habile machine à déglinguer) face à un système de valeurs et un ensemble de situations posées comme aliénantes. À plusieurs d'entre eux la dérision tient lieu de toute réussite, ce en quoi leurs ouvrages entretiennent les pratiques qu' ils semblent condamner tant elle est devenue l'une des voix officielles de la société du spectacle, sa doublure autorisée. C'est l'une des ambiguïtés de cette littérature, dont on souhaite qu'elle ne devienne pas (trop vite) ses apories. Autour des mêmes problématiques, on lira a contrario un texte de Nicole Caligaris intitulé L'Os $d u$ doute ${ }^{\text {II }}$, qui mène une recherche stimulante sur la vulgarisation de nouvelles parlures entrepreneuriales et les nouveaux mots d'ordre qui en résultent - recherche qui saisit à la racine, mais détourne, trafique et télescope les consignes mentales, les injonctions idéologiques larvées propres au système économique actuel.

Ces différents parcours montrent aussi combien on ne saurait établir de césure entre littérature moderne et littérature au présent, là où le tournant des années 1980 marque surtout un changement de dominantes sans exclusive (le passage de la tentation d'un art pleinement abstrait à celui d'un art qui calculerait pleinement sa capacité de figuration). On peut à cet égard suivre à la

6. Ibid., p. 48-5I.

7. Ibid., p. 40, 96 et 98 .

8. Fr. Beigbeder, 14 euros, Grasset, 2002.

9. J.-Ch. Masserra, Amour, gloire et Cac 40, POL, 1999.

IO. J. Mauche, La Loi des rendements décroissants, Seuil, 2007.

II. N. Caligaris, L'Os du doute, Verticales, 2006. 
trace, sur plus d'un siècle et dans un sens totalement réversible, les évolutions du personnage de roman, figure médiatrice par excellence du lien engagé avec l'idée de réalité - historique, psychique, événementielle - dont la contestation, souvent présentée comme la panacée de la modernité, n'a jamais eu valeur d'annihilation ni même revêtu une quelconque homogénéité. Quelle que soit la période du vingtième siècle envisagée - et ceci constitue aussi un appel à penser par synchronie l'histoire littéraire moderne - la composition du personnage de roman hésite entre le profil psychoréaliste, chez François Mauriac comme Nina Bouraoui, le soliloque désincarné, chez Louis-René des Forêts comme Éric Chevillard, l'énonciation à la dérive, chez Samuel Beckett comme Jacques Serena, les discours socialement ciblés, chez Proust comme Lydie Salvayre, les clichés localisés, ou langues de bois, chez Jean Tardieu comme Jean Echenoz. On ne saurait donc cautionner une lecture de l'histoire littéraire qui oppose une modernité répressive de tout rapport au réel à une actualité qui s'adonne de nouveau à son culte. L'idée de "retour» établit en amont une ligne de démarcation entre pratiques psychoréalistes et pratiques textualistes qui ont constitué l'avers et le revers d'un seul et même phénomène de reconnaissance aujourd'hui révolu : la toute-puissance du réel, son évidence complexe, affirmées tantôt sur le mode positiviste de la représentation mimétique, tantôt sur le mode négativiste de l'abstraction spéculaire. Un rapport entropique au réel a succédé à ces approches contraires qui ont motivé jusque dans les années 1970 les écritures de sa certification figurative autant que celles, symétriquement inversées, de sa dénégation autotélique.

Dernière série d'exemples : certaines œuvres travaillent le réel sur le mode de la remédiation. Entendons par là qu'elles recyclent un certain nombre d'imageries formant une conscience diffuse du réel, entre des automatismes quotidiens, des stéréotypes d'époque et des modèles culturels. Ce recyclage tient lieu à lui seul de mise à distance et de charge d'intelligibilité nouvelle. On rapprochera dans cette perspective - et dans cette perspective seulement quelques tentatives récentes : Microfictions ${ }^{12}$ de Régis Jauffret, qui brasse un millier d'histoires courtes déclinant, comme un spectacle d'ombres chinoises à peine esquissé les mille et une facettes d'une humanité des temps présents, à profil bas; Doggy bag de Philippe Djian, qui récupère, entre pastiche et revitalisation, les standards de certaines séries télévisées américaines soap opera pour actualiser les figures types (séduction, vengeance, inhibition, haine) et les structures-pivots (famille, loisirs, métier, opinions, modes) dans lesquelles elles s'inscrivent traditionnellement ${ }^{13}$; Les Éphémères, création collective du

I2. R. Jauffret, Microfictions, Gallimard, 2006.

I3. Ph. Djian, Doggy bag : plusieurs tomes parus depuis 2005 chez Julliard. 
Théâtre du Soleil, dirigée et mise en scène par Ariane Mnouchkine, qui sollicite, depuis un dispositif théâtral de tréteaux mobiles, une centaine de scènes d'intimité dans lesquelles, loin de tout traitement épique, le banal devient pourtant le capital, parce qu'il signe dans le temps (les trois dernières décennies) une histoire des mentalités communes et des comportements usuels - rupture du couple, mort d'une mère, élucidation d'un secret de famille, dérive de la folie douce, rapport à l'enfant.

Expérimentations en douceur menées sur quelques objets et situations constitutifs de notre rapport actuel au réel; jeu avec les discours dominants, leurs usages et leurs fins; effets de remédiations, tels sont quelques traitements romanesques des mythologies ordinaires contemporaines. Par-delà ces exemples, ce sont trois lieux communs qui encombrent parfois le discours critique sur la littérature au présent que j'ai aussi souhaité mettre en évidence. Premièrement, l'idée qu'il y aurait une permanence de fond du réel, que ce réel pourrait donc revenir en état après avoir été contourné ou que le roman pourrait y retourner à l'identique, pour peu que le romancier en balise la surface de quelques paillettes d'époque alors même que les modèles culturels et scientifiques permettant de l'appréhender se sont depuis un demi-siècle profondément modifiés. C'est autour de l'équilibre délicat entre la part des variables culturelles et celle des invariants anthropologiques que les ouvres majeures de notre temps définissent leur rapport au réel. Deuxièmement, l'idée que la littérature moderne ait pu déserter le réel parce qu'elle s'est engagée dans des voies expérimentales et dans un refus de toute figuration frontale, ce qui semble une vision pour le moins schématique de l'histoire (du) littéraire ${ }^{14}$. Troisièmement, dans l'un et l'autre cas, un déni de modernité qui tourne à sa détestation, ne rend pas compte des linéaments entre modernité historique et contemporanéité au présent, et n’est pas sans recouvrir quelque prurit réactionnaire.

Si certains auteurs s'inscrivent dans la lignée des Mythologies tout en marquant l'écart de fait et de temps qui les en sépare, c'est en leur qualité d'herméneutes par le doute. Ils s'essaient à décrypter des signes, à les constituer en ensembles susceptibles de faire sens, à induire quelques questions d'éthique à côté de tout esprit de système, sans opposer à une idéologie dominante une contre-idéologie elle-même totalisée en vue de quelque fin certifiée. Le devenirroman des Mythologies recouvre peut-être le développement de ce point de

I4. Du moins une vision qui, en se limitant à une double fantasmatique contraire, celle, révolue, d'une modernité négativiste, celle, actuelle, d'une postmodernité académique, occulte l'échelle même des nuances et les infinies graduations dont est faite une littérature en temps réel. 


\section{Recherches \& Travaux $-\mathrm{n}^{\circ} 77$}

latence qui caractérise le texte de Barthes : un accomplissement en fiction, marquant l'atomisation de tout appareillage systématique, qu'il soit sémiotique ou politique, comme happé dans le vertige abyssal du mythologique; un frottement des discours et un flottement des valeurs, qui génèrent par leur seule dynamique une démarche critique, une tonalité ludique, un arrière-plan mélancolique. 